\title{
Activity in the human brain predicting differential heart rate responses to emotional facial expressions
}

\author{
Hugo D. Critchley, a,b,c,* Pia Rotshtein, ${ }^{\mathrm{a}}$ Yoko Nagai, ${ }^{\mathrm{d}}$ John O’Doherty, ${ }^{\mathrm{a}}$ \\ Christopher J. Mathias, ${ }^{\mathrm{c}}$ and Raymond J. Dolan ${ }^{\mathrm{a}, \mathrm{b}}$ \\ a Wellcome Department of Imaging Neuroscience, Institute of Neurology, UCL, 12 Queen Square, London WC1N 3BG, UK \\ ${ }^{\mathrm{b}}$ Institute of Cognitive Neuroscience, UCL, 17 Queen Square, London, UK \\ ${ }^{\mathrm{c}}$ Autonomic Unit, National Hospital for Neurology and Neurosurgery and Neurovascular Medicine Unit, \\ St. Mary's Hospital Imperial, London, UK \\ ${ }^{\mathrm{d}}$ Institute of Neurology, UCL, Queen Square, London, UK
}

Received 26 April 2004; revised 23 September 2004; accepted 8 October 2004

Available online 24 November 2004

The James-Lange theory of emotion proposes that automatically generated bodily reactions not only color subjective emotional experience of stimuli, but also necessitate a mechanism by which these bodily reactions are differentially generated to reflect stimulus quality. To examine this putative mechanism, we simultaneously measured brain activity and heart rate to identify regions where neural activity predicted the magnitude of heart rate responses to emotional facial expressions. Using a forewarned reaction time task, we showed that orienting heart rate acceleration to emotional face stimuli was modulated as a function of the emotion depicted. The magnitude of evoked heart rate increase, both across the stimulus set and within each emotion category, was predicted by level of activity within a matrix of interconnected brain regions, including amygdala, insula, anterior cingulate, and brainstem. We suggest that these regions provide a substrate for translating visual perception of emotional facial expression into differential cardiac responses and thereby represent an interface for selective generation of visceral reactions that contribute to the embodied component of emotional reaction.

(c) 2004 Elsevier Inc. All rights reserved.

Keywords: Autonomic; Heart rate; Emotion; Facial expression; Functional magnetic resonance imaging

\section{Introduction}

The brain's representation of bodily responses and states of peripheral arousal has been proposed as a substrate for emotional feeling states (James, 1894). Emotive stimuli are proposed to elicit subjective emotions via the intermediary of autonomic, visceral,

\footnotetext{
* Corresponding author. Fax: +44 2078131420.

E-mail address: h.critchley@fil.ion.ucl.ac.uk (H.D. Critchley).

Available online on ScienceDirect (www.sciencedirect.com).
}

and skeletomotor responses (Damasio, 1994; Dolan, 2002; James, 1894; Lang et al., 1998).

Thus, fear is suggested as the experiential consequence of running from a bear (James, 1894). A prediction of this model is that emotive stimuli generate differential patterns of bodily arousal, which consequently feedback to the brain, so as to color subjective feelings.

Controversy exists over the degree to which bodily arousal, as for example, mediated via the autonomic nervous system, reflects the breadth of emotional experience. The Cannon-Bard theory of emotion argued that bodily arousal states are too undifferentiated to contribute to central emotional processing as anything more than epiphenomena (Cannon, 1927). Schachter and Singer (1962) similarly argued that a cognitive context is required to attribute arousal to any particular quality of emotional experience. The James-Lange theory embodies the concept that different emotional stimuli engender different bodily responses: that the sight of a bear gives rise to the feeling of fear because it excites a different bodily response to that engendered by nonfear stimuli such as the sight of a rabbit. The Cannon-Bard and Schachter-Singer theories of emotion do not predict that emotion quality is consequent upon a differential bodily response and therefore do not predict emotionspecific patterns of arousal. Nevertheless, induction of different emotional states produces different response patterns across autonomic axes (Collet et al., 1997; Levenson et al., 1990), and conscious or unconscious appraisal of facial expressions produces differential covert electromyographic responses in facial musculature (Dimberg et al., 2000; Surakka and Hietanen, 1998), suggesting that central appraisal of emotion-eliciting stimuli automatically generates differential bodily states.

Emotional facial expressions are salient communicative stimuli that influence behavior and provide powerful probes for investigating emotion-related neural mechanisms (Barrera and Maurer, 1981; Blair, 2003; Ekman, 1993). Specific brain regions, including fusiform and superior temporal cortices, are implicated in selective 
processing of facial characteristics such as identity, familiarity, and eye-gaze direction (Kanwisher et al., 1997; Kuskowski and Pardo, 1999; Puce et al., 1998). Superior temporal lobe regions are implicated, in both animal and humans studies, in processing movements and features underlying explicit discrimination of facial expressions (Hasselmo et al., 1989; Puce et al., 1998), but the extent to which emotional facial expressions are processed further along "labeled lines," with separate neural systems accounting for different emotions, is uncertain.

The amygdala is strongly implicated in processing threat and fear stimuli in animals and humans (Adolphs et al., 1994; Buchel et al., 1998; Hamann et al., 1996; LeDoux, 1996; LeDoux et al., 1998; Morris et al., 1996, 1997, 1998, 2002) but is also implicated in processing positive value of stimuli (Baylis and Gaffan, 1991; Breiter et al., 1996; Gottfried et al., 2003; Hamann and Mao, 2002; O'Doherty et al., 2002; Schwartz et al., 2003). Amygdala responses correlate with emotional intensity for positive and negative facial expressions during explicit or incidental processing (Winston et al., 2003), suggesting that the amygdala nonspecifically encodes emotionality. The central amygdala nucleus is implicated as a generator of autonomic arousal responses to threat stimuli (Iwata et al., 1987; LeDoux et al., 1998). Amygdala responses correlate with evoked sympathetic arousal to fear stimuli, anticipated threat, and subliminal threat (Buchel et al., 1998; Morris et al., 1997; Phelps et al., 2001; Williams et al., 2001). Experiments using relatively nonemotional stimuli, however, implicate regions of dorsal anterior cingulate in generating integrated autonomic (sympathetic) arousal responses (Critchley et al., 2000a, 2003), whereas an inverse relationship is observed between sympathetic arousal and subgenual cingulate and medial orbitofrontal activity, suggesting that this latter region may have an antisympathetic or parasympathetic role (Nagai et al., 2004). Within insula cortex, an anterior ventral region has been implicated in selective encoding of disgust stimuli and representation of facial expressions of disgust (Phillips et al., 1997). Adjacent regions of midand anterior insula cortex are also implicated in mapping interoceptive bodily states, where activity correlates with both evoked arousal responses (Critchley et al., 2000a,b, 2001, 2003) and interoceptive awareness (Critchley et al., 2004). One suggestion from these studies is that activity in amygdala and parts of insula cortex reflects processing of emotion quality, as well as obligatory generation and remapping of emotionally evoked bodily responses.

The goal of this study was to identify brain regions supporting a translation of perceived emotionality into autonomic bodily responses. While existence of such a mechanism is consistent with the James-Lange theory of emotion, we did not set out to prove the exclusive validity of a Jamesian account for all emotional processing. Our approach was to identify regional brain activity that predicted evoked heart rate responses to emotional facial expressions. We first demonstrated that perception of emotional facial expressions evoked differential changes in heart rate response according to emotion category. Activity in a discrete set of brain regions correlated with the magnitude of this heart rate change, predicting the cardiac response to face stimuli representing different emotion categories, as well as the cardiac response within an individual emotion category. Our findings indicate that visceral changes evoked by emotional stimuli are tightly coupled with activity within the amygdala, insula, anterior cingulate, and brainstem.

\section{Materials and methods}

Subjects and task design

Fifteen healthy right-handed volunteers English-speaking volunteers of British and European ancestry (mean age $32 \pm 8$ years; six males, nine females) gave informed written consent to participate in an functional magnetic resonance imaging (fMRI) scanning study approved by the local Ethics Committee. Subjects were all in full-time employment and further education and were screened by medical/psychiatric interview and questionnaire to exclude history or evidence of neurological, medical, or psychological disorder including substance misuse.

Critical to the experimental design was the use of a forewarned reaction time task, in which a preparatory orienting cue precedes a fixed delay interval ending with an imperative cue for a reaction time response. Such tasks produce a transient acceleration in heart rate followed by heart rate deceleration prior to the response (Frith and Allen, 1983; Jennings et al., 2002). In the present study, we used emotional faces as orienting cues and measured the influence of these faces on heart rate acceleration. Subsequent reaction-time responses were forced-choice judgments of the facial expression portrayed in the orienting cue. This design allowed us to examine regional activity during processing of emotional faces and to test for activity predicting changes in the magnitude of heart rate response induced by the stimuli.

Each subject performed one session of 80 randomized trials of the emotional forewarned reaction time task. A trial began with 1-s presentation of a photograph image depicting a happy, angry, sad, or disgusted facial expression. Then, a predictable (7 s) interval (blank screen with fixation cross) preceded a visual prompt to press one of four buttons according to category of the facial emotion. Subjects were required to make this judgement as rapid as possible (reaction-time response). Intertrial intervals were jittered with a mean of $7.4 \pm 1 \mathrm{~s}$ (Fig. 1). The face stimuli were taken from a standardized face database (The Karolinska Directed Emotional Faces-KDEF; Lundqvist et al., 1998). Selection of faces to control for expression intensity and quality and ensure near $100 \%$ recognition accuracy was determined at behavioral piloting in nine control subjects prior to the study of Winston et al. (2004). The choice of happy, angry, sad, and disgusted expressions among putative "standard" emotions was determined within constraints of task length to embody dimensions of valence, motivation (approach/withdrawal), and autonomic response (e.g., Ekman et al., 1983). Neutral faces were excluded since on pilot runs, they tended to evoke no differential autonomic response and were not critical to interpretation of study results. Fearful faces were not included as neural correlates of fear expressions processing have been widely reported within the literature. Button-press responses were made with fingers of the right hand during the scanning using an in-house-devised MRI compatible four-choice button box.

\section{fMRI data acquisition}

Sequential T2*-weighted echoplanar images (Siemens Sonata, 1.5-T, 28 slices, $3.5 \mathrm{~mm}$ thick, TE $50 \mathrm{~ms}, \mathrm{TR}_{\mathrm{vol}} 2.52 \mathrm{~s}$; voxel size $1 \times 1 \times 3.5 \mathrm{~mm})$ were acquired for blood oxygenation leveldependent (BOLD) contrast. Slices were acquired to provide whole brain coverage in an oblique orientation $30^{\circ}$ to the anteriorposterior commissural line to optimize sensitivity to orbitofrontal cortex and medial temporal lobes (Deichmann et al., 2003). Images 
A) Experimental task with heart rate monitoring (pulse oximetry)

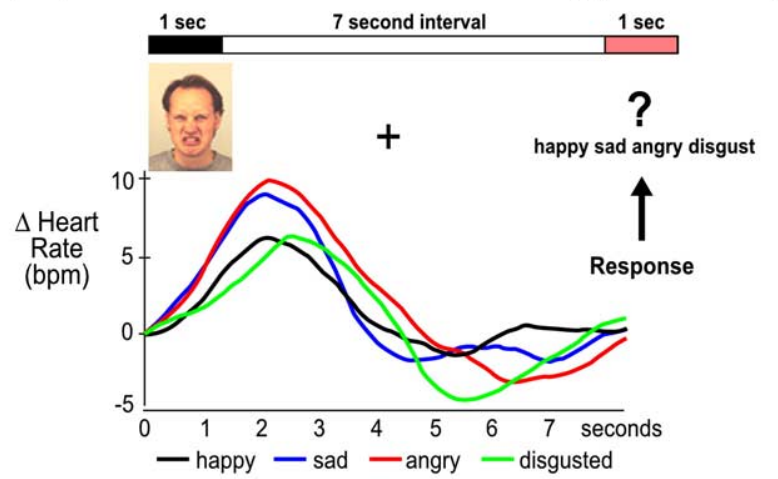

B) Normalised heart rate responses to emotional expressions

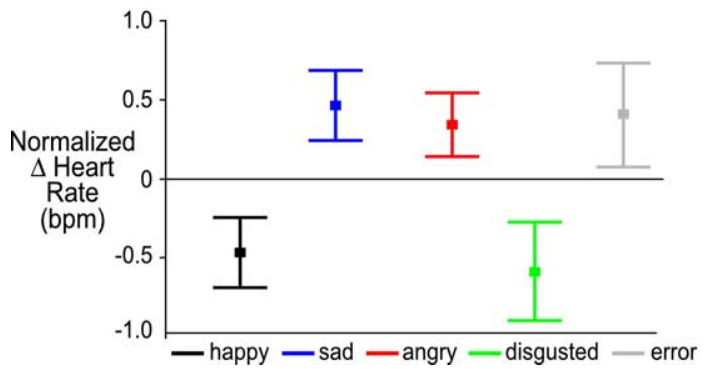

Fig. 1. Experimental design and cardiac responses. (A) Subjects performed 80 trials of an emotional forewarned reaction time task. Each trial began with a 1-s presentation of a face stimulus depicting a happy, sad, angry, or disgusted facial expression. The subject maintained fixation over an ensuing 7-s interval then made a cued four-choice, reaction-time, buttonpress judgement of the facial emotion. There then followed a variable intertrial interval of mean duration $7.5 \mathrm{~s}$. This trial procedure is associated with and orienting cardiac acceleration to the emotional face, followed by cardiac deceleration in anticipation of the cued motor response. The figure illustrates diagrammatically an experimental trial and, beneath this, average heart rate responses (interpolated pulse oximetry data, zeroed at trial onset) of one subject during trials judged to depict happy (black), sad (blue), angry (red), and disgusted (green) facial expressions. This color convention is used in other figures. (B) The peak heart rate in the first $4 \mathrm{~s}$ of each trial, relative to the trial onset, was computed and used in the analyses of functional imaging data. Across subjects, different emotional judgements were associated with different magnitude of heart rate increase. Compared to the average response, facial expressions judged as happy or disgusted were associated with a relative blunting of heart rate, whereas there was a relative increase in heart rate increase evoked by sad and angry facial expressions. Error responses (where there was a face wrongly categorized, depicted in gray) also produced a relative enhancement of heart rate increase. Group data in the evoked change in heart rate (mean $\pm \mathrm{SE}$ ) are shown, representing changes from an average heart rate of 66.1 beats per minute across subjects (range $54-76$ beats per minute).

were reconstructed with reference to a trajectory calibration scan, limiting Nyquist ghosting (Josephs et al., 2000). Head movement was minimized during scanning by comfortable external head restraint and subsequently corrected for using realignment and unwarping functions in the preprocessing prior to data analyses (see below). Five hundred whole-brain images were obtained over $21 \mathrm{~min}$. The first six echoplanar volumes were not analyzed to allow for T1-equilibration effects. A T1-weighted structural image was also obtained for each subject to facilitate anatomical description of individual functional activity after coregistration with fMRI data.
During functional imaging, we simultaneously recorded heart rate using pulse oximetry from the left index finger (Nonin 8600 Pulse Oximeter, Nonin Medical, Inc., N. Plymouth, MN, USA). Timings of each finger pulse were logged with slice synchronization data pulses from the scanner using CED 1401 data acquisition unit and Spike 3 software (CED, Cambridge Electronic Design Limited, Cambridge, UK).

\section{Analysis of heart rate measures}

The waveform of each subject's pulse, obtained using pulse oximetry, was recorded at $80 \mathrm{~Hz}$ throughout the imaging study. These data were then interpolated at $100 \mathrm{~Hz}$ and resampled to derive stimulus event-related measures of the peak heart rate change over the 4-s period following face stimulus onset. These event-related measures were used as parametric modulators of interest in the neuroimaging analyses.

\section{fMRI data analysis}

We used SPM2 (http://www.fil.ion.ucl.ac.uk/spm/spm2.html) on a Matlab platform (Mathwork, IL) to analyze fMRI data. Scans for each subject were realigned (motion-corrected), unwarped (correcting in part for distortions attributable to head movement), and corrected for differences in timing of each slice. Scan volumes were spatially transformed to standard stereotaxic space (with respect to the Montreal Neurologic Institute coordinate system) and smoothed (Gaussian kernel full-width half-maximum, $8 \mathrm{~mm}$ ) prior to analysis. Design matrices were constructed for each subject to model presentation of face stimuli as events [delta functions convolved with a canonical hemodynamic response function (HRF)], and duration of the whole trial up to response as an 8-s boxcar epoch, both convolved with HRF. Heart rate changes were treated as parametric modulators of the face stimulus events to identify activity occurring at the time of face processing that correlated with the subsequent change in heart rate. The first analytic design modeled all face events (and epochs) together to test for activity predicting heart rate acceleration across all emotional face stimuli. However, because heart rate change was related to the category of emotion presented, this first analysis is potentially confounded by identifying brain regions responsive to an emotion type but not predictive of bodily response to the stimuli. We therefore conducted a second analysis that modeled five-event and -epoch trial types, representing trial in which happy, sad, angry, and disgusted facial expressions were correctly identified and the error trials where the expression was incorrectly identified. The magnitude of heart rate increase was entered as a parametric modulator of event-related activity for each of the five event types, thereby allowing us to test for activity predicting the magnitude of heart rate response separately within each emotion category. This approach removes potential confounds of overall differences in evoked heart rate between emotion categories. Moreover, by modeling epoch-related activity within these individual designs, examination of event-related activity enables detection of transient primary responses to the stimuli while controlling for sustained activity relating to emotional appraisal and attention to emotional content. In individual subject analyses, nonsphericity attributable to serial correlations in the fMRI time series was modeled using an autoregressive process with white noise. fMRI time series were decomposed into experimental effects of interest (embodied in the design matrix) and errors representing 
Table 1

Activity correlated with heart rate change evoked by viewing emotional face stimuli across emotion types

\begin{tabular}{lllc}
\hline Brain region & Side & Coordinates & $Z$ score \\
\hline Medial fusiform/lingual gyrus & $\mathrm{L}$ & $-18,-64,-20$ & 11.3 \\
& $\mathrm{R}$ & $22,-66,-24$ & 10.3 \\
Insula/operculum & $\mathrm{L}$ & $-52,-2,16$ & 10.9 \\
& $\mathrm{R}$ & $40,-2,18$ & 8.81 \\
Dorsal anterior cingulate & $\mathrm{L}$ & $-10,-2,52$ & 10.1 \\
Intraparietal sulcus & $\mathrm{R}$ & $36,-40,40$ & 9.8 \\
Superior parietal lobule & $\mathrm{R}$ & $16,-72,46$ & 9.3 \\
Superior temporal sulcus & $\mathrm{L}$ & $46,-34,14$ & 7.9 \\
Anterior hippocampus & $\mathrm{L}$ & $-30,-8,-18$ & 6.8 \\
& $\mathrm{R}$ & $34,-8,20$ & 6.8 \\
Head of caudate & $\mathrm{R}$ & $10,6,-2$ & 6.9 \\
Amygdala/uncus & - & $22,8,-16$ & 6.3 \\
Pons & - & $4,-22,-24$ & 7.16 \\
\hline
\end{tabular}

fixed deterministic drifts (removed by high-pass filtering) and stochastic short-term correlations conforming to an $\mathrm{AR}(1)$ process (http://www.fil.ion.ucl.ac.uk/spm/spm2.html; Friston et al., 2002).

Second-level (random effects) analyses were performed as a $t$ test of event-related activity correlating with heart rate acceleration across all stimuli and as an ANOVA of event-related activity and relationship to heart rate increase for each emotion type (using sphericity correction for within-subject repeated measures). In the first analysis, we tested for regional activity, at the time of face events, correlating with observed magnitude of subsequent heart rate change across all stimuli. In the second analysis, we examined the event-related activity reflecting the main effect of processing of emotional faces and the main effect of activity predictive of stimulus-induced heart rate increases. To test for possible confounding effects of gender on emotional processing or evoked cardiac responses, one version of this ANOVA included a gender-specific regressor. No significant modulation was attributable to gender, therefore subsequent analyses did not model gender. In group analyses, we used conjunction analyses across each emotion category to ensure that activity ascribed to face processing and activity predictive of evoked heart rate increases was common to all face categories and not driven by one emotion type alone. We also tested, using $F$ contrasts, for activity that reflected differential processing of face emotions. Thus, we tested for differential activity to events across emotion category (independent of within-category heart rate increases) and for activity differentially predicting heart rate increases to some but not all emotions. Threshold significance for random effects analysis was set at $P<0.05$ corrected for multiple comparisons using Family Wise Error (FWE). Anatomical location of activity was determined with reference to the atlas of Duvernoy (1999) from the site of peak voxel activity within a significant cluster (spatial smoothing has no effect on position), superimposed on normalized group-mean T1-weighted (structural) and EPI images.

\section{Results}

\section{Behavior and heart rate responses}

Subjects made on average a total of $11 \pm 3.5$ errors during the task, representing a misclassification of facial expressions on
$13.7 \%$ of trials. The most frequent errors were made in judging facial expressions of disgust (errors to disgust stimuli $7 \%$ of trials; anger $3 \%$; sadness $3 \%$; happiness $<1 \%$ ). There were no significant differences in reaction time between correct and incorrect judgments or responses to the different (correctly identified) emotional categories $[F(5,56)=0.27$, n.s. $]$.

Subjects' mean heart rate varied from 54 to 76 beats per minute (group mean $=66.1 \pm 6.9$ beats per minute). The mean evoked heart rate response to the face stimuli across subjects was an increase of $4.6 \pm 20.8$ beats per minute. Importantly, there was a significant effect of stimulus type and emotional category on the magnitude of heart rate increase evoked by the face stimuli $[F(4,70)=3.90, P<0.01]$ (Fig. 1). Correctly identified sad and angry faces, and faces presented on error trials, evoked significantly larger heart rate responses than presentations of happy or disgusted faces (Fig. 1). It is noteworthy that presentation of the most and least easily identified facial expressions (happiness and disgust) was associated with diminished magnitude of evoked heart rate increase, compared to presentation of sad and angry expressions. This observation indicates that heart rate responses did not represent a simple effect of recognition difficulty or of positive/negative emotional valence. Moreover, expressions of disgust that were misidentified as portraying sadness or anger evoked heart rate changes more typical of sadness and anger than disgust, as perhaps predicted by the James-Lange model. These observations indicate that some facial expressions can be differentiated psychophysiologically on the basis of evoked heart rate response. Thus, regional activity predicting heart rate increase is likely to contribute to the differential processing between different categories of emotional facial expressions that may be associated with differential arousal patterns across multiple autonomic axes.

Activity relating to heart rate change across emotional face stimuli

For each subject, we performed a correlational analysis of the evoked heart rate response to each stimulus and analyzed the group effects in a second-level $t$ test. Brain regions whose activity reflected evoked change in heart rate (at corrected significance) included bilateral insula opercular, dorsal anterior cingulate and right parietal cortices, bilateral anterior medial temporal lobe regions (hippocampus, amygdala, and uncus), and brainstem (Table 1). This approach is to some extent biased toward identifying brain areas more responsive to sad and angry, compared to happy and disgusted, facial expressions by virtue of the fact that the former engender larger heart rate responses. We therefore performed a further set of analyses to identify and dissociate brain areas supporting categorical processing of emotion type from brain regions predicting heart rate changes to emotional stimuli, while stringently controlling for the potential confound of emotion type.

Activity relating to categorical and autonomic responses to processing of emotional faces

In these subsequent analyses, we modeled presentation of face events (and error events) and evoked heart rate changes separately according to the emotion portrayed. This allowed us to examine responses to each different facial expression (while controlling for variations in "arousal" within an expression category). Additionally, it enabled us to test for regional activity correlating with heart rate increases within-category, while controlling for differences in the evoked arousal observed between expression categories. An $F$ 
Table 2

\begin{tabular}{|c|c|c|c|}
\hline Brain region & Side & Coordinates & $Z$ score \\
\hline \multicolumn{4}{|c|}{ a) Activity related to main effect of categorical processing of face expression (conjunction analysis of emotion types) } \\
\hline \multirow[t]{2}{*}{ Fusiform cortex } & $\mathrm{R}$ & $42,-60,-16$ & $>10$ \\
\hline & $\mathrm{L}$ & $-40,-60,-12$ & $>10$ \\
\hline \multirow[t]{3}{*}{ Dorsolateral prefrontal cortex } & $\mathrm{R}$ & $42,12,28$ & $>10$ \\
\hline & & $44,32,14$ & $>10$ \\
\hline & $\mathrm{L}$ & $-38,4,28$ & $>10$ \\
\hline \multirow[t]{2}{*}{ Anterior insula } & $\mathrm{L}$ & $-38,22,-6$ & $>10$ \\
\hline & $\mathrm{R}$ & $36,26,0$ & $>10$ \\
\hline Striate cortex & - & $2,-84,-4$ & $>10$ \\
\hline \multirow[t]{2}{*}{ Inferior parietal lobule } & $\mathrm{R}$ & $32,-52,48$ & $>10$ \\
\hline & $\mathrm{R}$ & $30,-48,46$ & 7.34 \\
\hline Superior temporal sulcus & $\mathrm{R}$ & $52,-42,12$ & $>10$ \\
\hline \multirow[t]{2}{*}{ Posterior hippocampus/parahippocampal gyrus } & $\mathrm{R}$ & $16,-34,-2$ & $>10$ \\
\hline & $\mathrm{L}$ & $-22,-30,-4$ & 7.63 \\
\hline Dorsomedial prefrontal/anterior cingulate & - & $-4,-8,56$ & $>10$ \\
\hline \multirow[t]{2}{*}{ Amygdala } & $\mathrm{R}$ & $18,-6,-20$ & 6.72 \\
\hline & $\mathrm{L}$ & $-16,-6,-18$ & 5.20 \\
\hline \multirow[t]{2}{*}{ Head of caudate ${ }^{a}$} & $\mathrm{R}$ & $10,12,-2$ & $5.81^{\mathrm{a}}$ \\
\hline & $\mathrm{L}$ & $-10,10,0$ & $5.49^{\mathrm{a}}$ \\
\hline \multicolumn{4}{|c|}{ b) Activity predictive of heart rate acceleration (conjunction analysis of correlations within each emotion type) } \\
\hline Midcingulate & - & $-8,-14,46$ & $>10$ \\
\hline \multirow[t]{3}{*}{ Insula } & $\mathrm{R}$ & $34,18,2$ & 7.56 \\
\hline & $\mathrm{R}$ & $30,-4,14$ & 6.31 \\
\hline & $\mathrm{L}$ & $-38,2,14$ & 6.35 \\
\hline \multirow[t]{3}{*}{ Amygdala } & $\mathrm{R}$ & $22,8,-16$ & 5.45 \\
\hline & $\mathrm{R}$ & $24,4,-18$ & 5.45 \\
\hline & $\mathrm{L}$ & $-30,0,-12$ & 5.55 \\
\hline \multirow[t]{4}{*}{ Lingual and fusiform gyri } & $\mathrm{R}$ & $22,-66,-24$ & 7.27 \\
\hline & $\mathrm{L}$ & $-17,-65,-15$ & 7.07 \\
\hline & $\mathrm{L}$ & $-10,-68,-8$ & 6.93 \\
\hline & $\mathrm{R}$ & $16,-70,-8$ & 6.52 \\
\hline Superior temporal cortex & $\mathrm{R}$ & $56,-16,16$ & 6.72 \\
\hline Cerebellum & $\mathrm{R}$ & $18,-46,-56$ & 6.32 \\
\hline \multirow[t]{2}{*}{ Thalamus } & $\mathrm{R}$ & $14,-16,0$ & 5.30 \\
\hline & $\mathrm{L}$ & $-14,-12,8$ & 5.73 \\
\hline Midbrain & $\mathrm{R}$ & $12,-20,-8$ & 5.30 \\
\hline \multirow[t]{2}{*}{ Brainstem (medulla) } & $\mathrm{L}$ & $-8,-28,-40$ & 5.35 \\
\hline & $\mathrm{L}$ & $-4,-32,-50$ & 5.12 \\
\hline
\end{tabular}

c) Areas activated by categorical face processing and whose activity correlated with heart rate acceleration (conjunction analysis of main effects)

Dorsal anterior cingulate

Anterior insula

Amygdala

Anterior hippocampus

Lingual and fusiform gyri

Cerebellar vermis

Head of caudate nucleus

Thalamus

Brainstem (pons)
Superior temporal cortex

$\begin{array}{llc}\text { L } & -8,-14,46 & >10 \\ \text { R } & 10,10,52 & >10 \\ \text { R } & 34,20,2 & >10 \\ \text { L } & -28,22,6 & >10 \\ \text { R } & 30,-4,14 & 6.31 \\ \text { L } & -38,2,14 & 6.35 \\ \text { R } & 30,6,-22 & 6.63 \\ \text { L } & -20,-2,-18 & 5.86 \\ \text { L } & -10,-12,-14 & 5.95 \\ \text { R } & 20,-62,-24 & >10 \\ \text { L } & -8,-70,6 & >10 \\ \text { L } & -28,-58,-18 & >10 \\ \text { R } & 30,-74,-14 & >10 \\ \text { R } & 58,-48,6 & 0.10 \\ \text { R } & 56,-48,14 & >10 \\ - & -2,-58,-44 & 5.86 \\ \text { R } & 10,8,2 & 6.21 \\ \text { L } & -12,6,4 & 5.70 \\ \text { R } & 8,-10,4 & 5.30 \\ \text { L } & -6,-12,2 & 5.91 \\ \text { L } & 2,-22,-22 & 5.25\end{array}$

${ }^{a}$ Head of caudate seen in main effect but not in conjunction analysis across emotions. 
test of a gender-specific regressor in this second-level ANOVA revealed no significant gender effects on processing of emotion category and emotion-specific heart rate responses. We first determined the main effect of emotional faces and correlations with heart rate across expression categories. In particular, we determined which brain regions predict differences in magnitude of heart rate response to emotional faces. In principle, such regions would show greater enhancement of activity to sad, angry, and error trials (associated with larger evoked cardiac response) than happy and disgust trials. A second question was whether any brain region responded differentially to emotional expressions when the autonomic response (evoked heart rate change) to the face stimuli was treated as a confound. A third question addressed was whether particular brain regions predicted increases in heart rate differentially to stimuli in some emotion categories, but not others.

The main effect of categorical processing of facial expressions was associated with enhanced activity within bilateral amygdala and head of caudate and bilateral striate, fusiform (ventral occipitotemporal), medial prefrontal/cingulate, anterior insular, and dorsolateral prefrontal cortices. We demonstrated, using conjunction analyses, that in majority of these regions (all except head of caudate), activity was enhanced to each individual emotional category (Table 2a).

Brain areas where activity correlated with the magnitude of heart rate acceleration following presentation of the face stimuli also included bilateral amygdala and bilateral early visual, fusiform, and lingual gyri and anterior insula cortices. Evoked increases in heart rate were also associated with enhanced activity in superior temporal (STG), midinsular, dorsal cingulate and cerebellar cortices, thalamus, pons, and medulla. We further showed using a conjunction analysis that, in each of these regions, the positive correlation between activity and heart rate increase was independent of emotion category (Table $2 b$ ). We found no regional activity that correlated negatively at threshold significance with heart rate changes. Only activity in a region of subgenual cingulate, extending posterior to the nucleus accumbens, showed a negative correlation with heart rate increase at an uncorrected threshold of $P<0.001$ (coordinates $-2,24,2, t=4.85, P=0.064$ corrected).

The conjunction of the main effect of face processing and positive correlation with evoked heart rate increase constrains the identification of brain regions to those that responded to emotional faces and showed enhanced activity correlated with evoked heart rate acceleration. This analysis identified early visual regions including V1, lingual gyrus, bilateral fusiform and lateral occipitotemporal cortices, bilateral superior parietal lobe, dorsolateral prefrontal cortex, bilateral anterior insula, midline dorsal anterior cingulate, and left hippocampus. Importantly, subcortical regions including bilateral amygdala, neostriatum (head of caudate), medial thalamus, and dorsal pons were also identified in this conjunction analysis (Fig. 2, Table 2c). It was inferred that activity in these regions, predicting the magnitude of evoked heart rate change to emotional faces, would be more enhanced to the initial presentation of sad and angry faces that evoke greater cardiac responses than happy and disgusted faces. This prediction was confirmed for amygdala and fusiform regions in analyses of categorical event-related responses (see Fig. 3) and in reanalyses of the data set (without accounting for evoked heart rate changes) for amygdala, occipitotemporal, fusiform, caudate, thalamic, and brainstem regions.

Although similar brain regions reflected categorical processing of face expression as well as predicted the magnitude of the evoked heart rate response, qualitative differences were noted in the location of peak activity, even within the same anatomical region. In the amygdala, activity responding to face events was located medial and posterior (in approximately the basolateral amygdala nucleus) compared to amygdala activity predicting heart rate, located anterior and lateral, consistent with the likely location of the central amygdala nucleus. In the ventral occipitotemporal region, activity related to face events was located lateral (in fusiform gyrus) to activity that correlated with heat rate response (including lingual gyrus). In addition, different regions of insula cortex were associated with processing faces and predicting heart rate increases, notably overlapping in right anterior insula (in a region implicated in conscious integration of interoceptive and exteroceptive information to support emotional awareness and feeling states (Critchley et al., 2002, 2004) (Fig. 4).

Finally, we tested whether any brain region differentiated between expression type in their responses to face events. Using an $F$ test, we examined all possible combinations of discrimination between emotional categories within 'face processing regions' identified as a main effect. Only right head of caudate and the left anterior insula reached criterion significance in this ANOVA. Activity in caudate nucleus showed increases to all negative expressions but decreases to happy faces. Left insula demonstrated enhanced activity to all expression types, greatest to presentation of sad faces and least to presentation of happy faces. In a further $F$ test, we found no significant event-related activity discriminating among negative expressions, based on categorical event-related responses, even at a threshold of $P<0.001$, uncorrected. We next tested for activity that differentially correlated with heart rate responses to expressions, that is, where activity predicted heart rate acceleration to some but not other categories of facial expression. We found no brain areas demonstrating an emotion-specific correlation between activity and evoked heart rate, even when we constrained our search to regions showing a main effect of correlations with evoked heart rate changes. We acknowledge, however, that we applied very stringent threshold criteria to our data analyses and may have been insensitive to some emotionspecific regions, for example, ventral insula cortex that may integrate parasympathetic cardiac responses with selective processing of disgust.

\section{Discussion}

Activity in a matrix of brain regions predicts the magnitude of cardiac response to visually presented emotional facial expressions. Relative increases in heart rate response were observed to sad and angry facial expressions and relative decreases to happy and disgusted facial expressions, indicating that specific brain regions may support differential processing of sad and angry from happy and disgusted expressions.

A central role is accorded to the amygdala in engendering differential visceral responses to emotional stimuli. Animal studies demonstrate that amygdala activity (particularly in the central nucleus (LeDoux et al., 1998)) initiates autonomic responses to salient stimuli. In human neuroimaging studies, this has been indexed in correlations between peripheral arousal and amygdala responses to emotive stimuli (Buchel et al., 1998; Critchley et al., 2002; Morris et al., 1997; Phelps et al., 2001, Williams et al., 2001). The emphasis in these earlier studies has been the processing of fear and threat stimuli. However, amygdala activity is also enhanced by 


\section{A) Face-responsive brain regions predicting heart rate response magnitude}
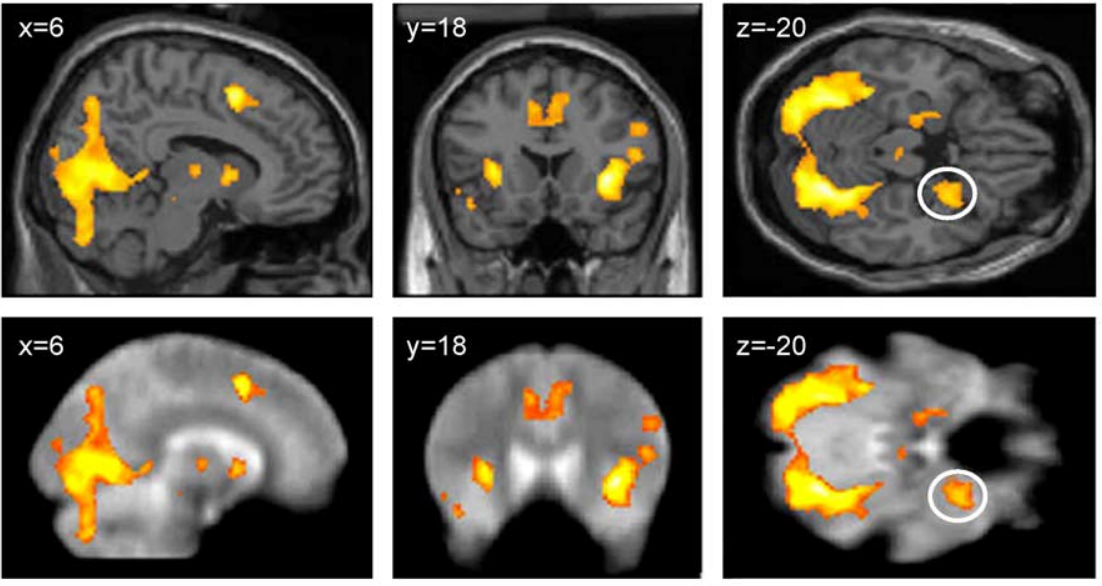

B) Amygdala: Group parameter estimates for correlations with heart rate response

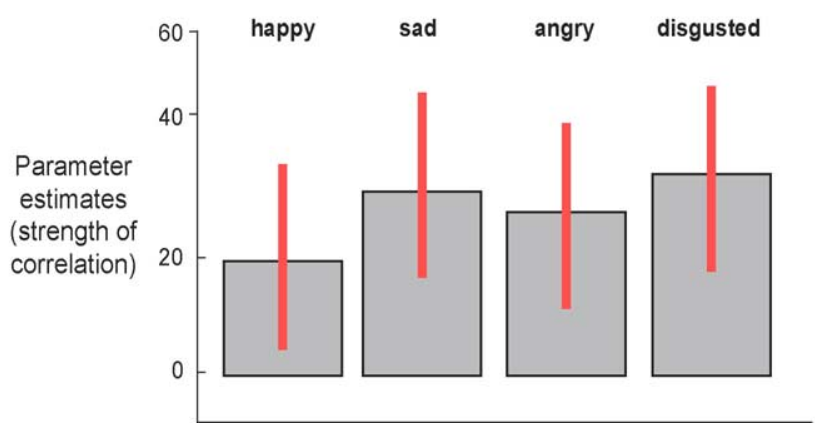

Fig. 2. Brain regions predicting heart rate response to faces. Activity in a set of brain regions correlated with enhancement of orienting heart rate acceleration induced by emotional face stimuli. Importantly, these regions were identified by correlations within each emotional category, thus differences in between emotion types did not contribute to this analysis. (A) The upper panel shows group activity predicting heart rate increases evoked by the stimuli (main effect of heart rate acceleration) mapped on sagittal, coronal, axial, and sections of a template brain. Coordinates in mm and laterality are given in section. Beneath is the same data mapped onto a mean echoplanar T2*-weighted image derived from all 15 subjects to highlight the field of view of the functional dataset including regions of signal dropout (that notably did not effect the amygdala region). Activity within the (right) amygdala is circled, yet the figure also demonstrates occipitotemporal, lingual, thalamic, caudate, dorsal cingulate, posterior insula, and medullary activities predicting heart rate response to the faces. (B) Parameter estimates of group amygdala activity correlating with heart rate increases. In our first- and second-level analyses, heart rate increases were modeled within emotion category. This figure illustrates that for each emotion, there were significant positive correlations between right amygdala activity [highlighted in (A) above] and heart rate for each emotion type, reflected in mean parameter estimates $+95 \%$ confidence limits at a second-level group analysis.

salient positive stimuli (Breiter et al., 1996; Gottfried et al., 2003; O'Doherty et al., 2002) and correlates with ratings of emotional intensity across different emotional facial expressions (Winston et al., 2003). Our own findings parallel these observations, in so far as amygdala activity predicted heart rate increases (an independent index of emotional arousal) evoked by face stimuli within each category of emotion. However, sad and angry expressions elicited on average greater heart rate increases than happy and disgusted facial expressions. In this regard, a greater amygdala response will be seen to sad and angry, compared to happy and disgusted, expressions. Our findings also point to dissociation of regions within the amygdala. Animal experiments highlight the role of the central nucleus of the amygdala in mediating peripheral autonomic responses induced by emotive stimuli (LeDoux et al., 1998). Regions such as the basolateral amygdala contribute to associative motivational learning (of both positive and negative associations (LeDoux, 1996). In our study, the location of amygdala activity that predicted heart rate acceleration was more anterior and lateral (in the region of the central nucleus) than activity related solely to the main effect of processing emotional face stimuli.
We also observed that the heart rate responses induced by emotional face stimuli correlated with activity in face-sensitive visual cortical areas, that is, fusiform and adjacent lingual gyrus in the ventral occipitotemporal region (Kanwisher et al., 1997; Kuskowski and Pardo, 1999), and superior temporal cortex (Puce et al., 1998). It is likely that the correlation between event-related activity in these visual regions and evoked heart rate responses results from a common origin in the amygdala. Amygdala activity not only initiates peripheral autonomic responses but provides modulatory enhancement to cortical regions processing salient stimuli (Morris et al., 1997, 1998). The ventral occipitotemporal visual cortical regions predicting heart rate acceleration overlapped with fusiform regions including "face areas" (Kanwisher et al., 1997; Kuskowski and Pardo, 1999). Activity in fusiform cortex may be modulated by covert or overt attention, emotionality (where the amygdala connectivity may facilitate to fusiform activity enhancement), and even covaries with fluctuations in sympathetic arousal (Critchley et al., 2000a,b; Morris et al., 1997, 1998; Vuilleumier et al., 2001). However, the activity we observed in the more medial lingual gyral region showed even stronger correlations 


\section{Amygdala activity: Correlations with heart rate change in single subject}

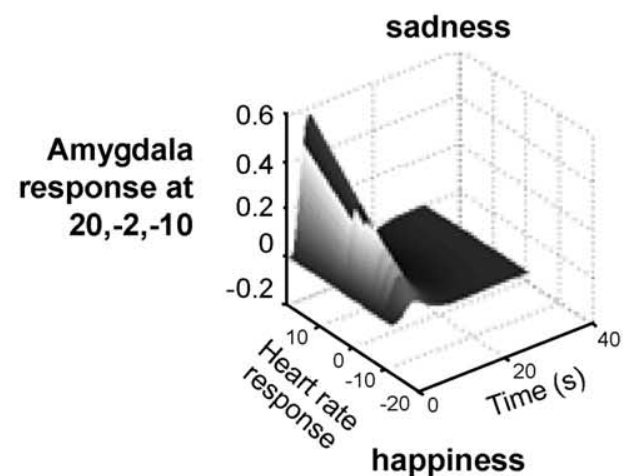

Response at 5.2 seconds
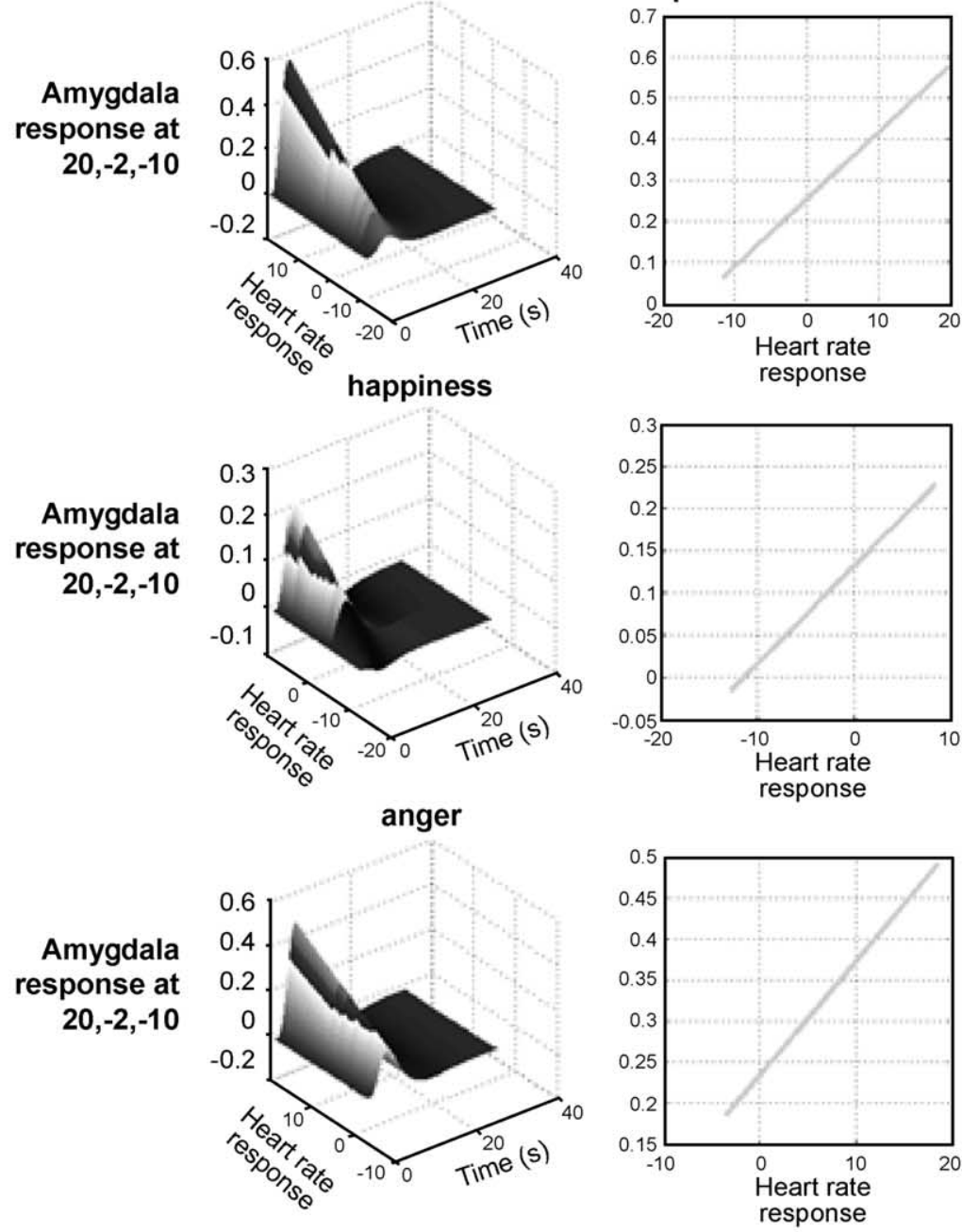

disgust
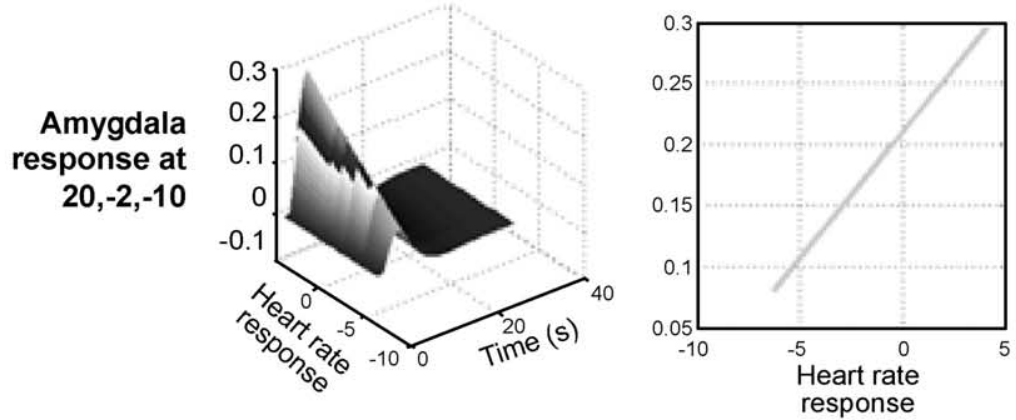

Fig. 3. Single subject data illustrating the correlational relationship between amygdala activity and expression-evoked heart rate changes. Event-related adjusted data from the right amygdala $(20,-2,-10)$ are plotted against the stimulus-evoked change in heart rate following stimulus presentation and as a function of peristimulus time in three-dimensional plots for sad, happy, angry, and disgusted face stimuli. The same data are plotted in two-dimensional graph illustrating BOLD activity at the approximate peak of the hemodynamic response (5.2. s after stimulus onset). Similar findings were replicated in each subject. While this figure serves to illustrate the correlations between amygdala activity and predicted cardiac response, as in Fig. 2, it also illustrates emotion-related differences in the actual magnitude of activity and cardiac responses which were larger to angry and sad face stimuli than to happy and disgusted stimuli.

with stimulus-induced heart rate responses. We note that activity in this region has been reported to covary with mood-induced states where heart rate is raised, for example, a strong subjective experience of grief (Gundel et al., 2003; Kuniecki et al., 2003).
Our observation of activity, correlated to evoked heart rate, in dorsal cingulate and insula provides further evidence for the role of these cortical brain regions in cardiovascular autonomic control. Previous studies report activity in both regions correlating with 


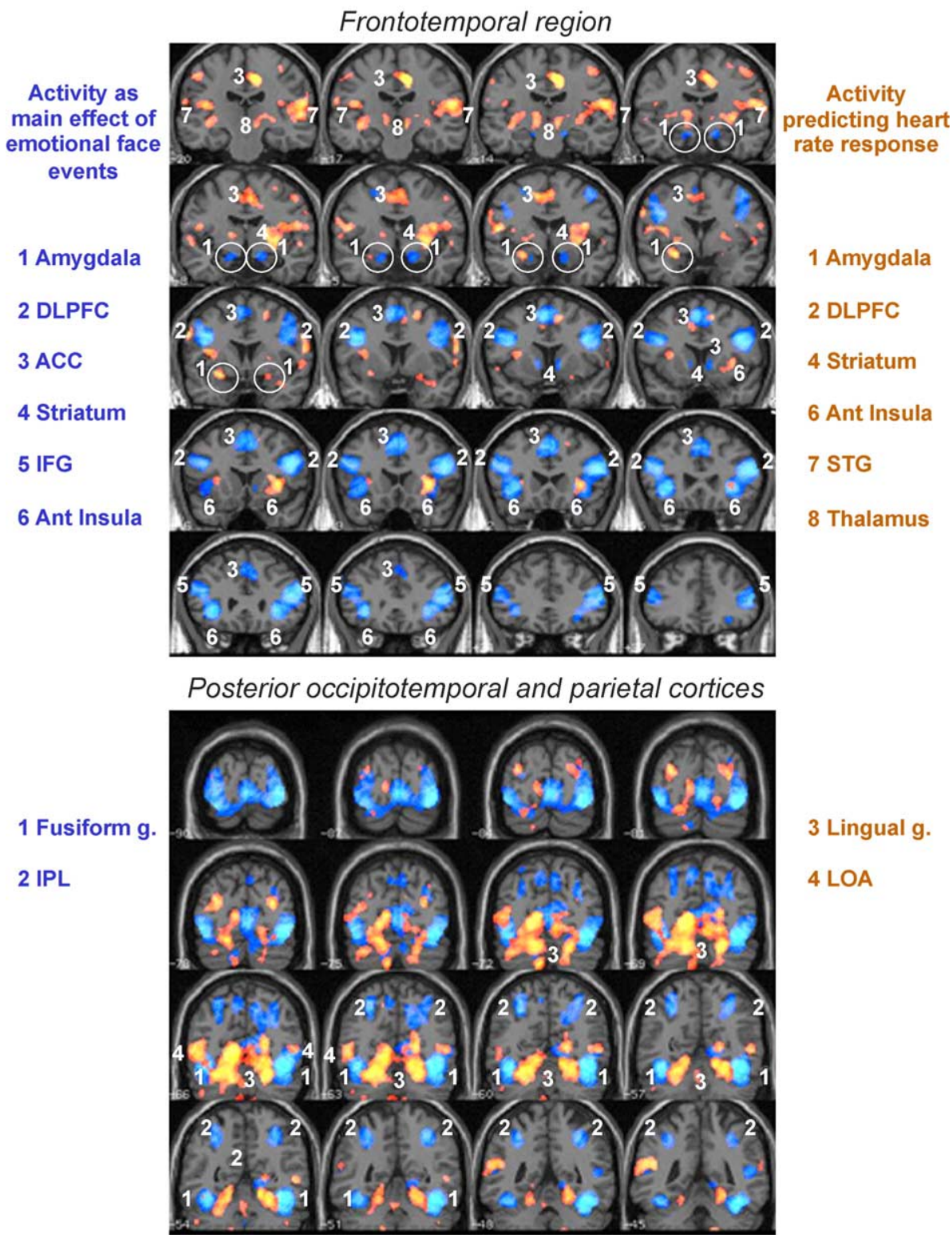

Fig. 4. Distribution of activity reflecting main effect of face processing and predicting heart rate response to faces. Group data are plotted on coronal sections of a template brain to illustrate the relative distribution of activity attributable to face events (blue) and predictive of heart rate responses (orange). A selection of brain regions is labeled, and the amygdala region is circled to highlight this area. Noteworthy are differences between event- and heart rate-related responses within the same and neighboring anatomical regions; for example, heart rate increases are predicted by activity in anterior lateral amygdala and lingual gyrus, in contrast to event-related activity in posterior amygdala and fusiform gyrus.

sympathetic influences on heart rate and other indices of autonomic arousal (Critchley et al., 2000a,b, 2001, 2003). Both regions reciprocally connect to brainstem and hypothalamic autonomic nuclei and amygdala (Barbas et al., 2003) and are activated during states of autonomic arousal and during evaluative emotional processing. In contrast to the role of dorsal cingulate in generating efferent arousal responses (Critchley et al., 2000a,b, 2003), anterior insula cortex is implicated strongly in afferent sensory representation of bodily arousal states (Critchley et al., 2001, 2002). This right anterior insula in particular is implicated in mediating conscious interoceptive awareness underlying feeling states (Critchley et al., 2004). Our observations of anterior insular activity correlating with evoked heart rate increases are likely to represent an integration of external emotional information with peripheral states of arousal, as noted in earlier investigations (Critchley et al., 2003, 2004). Importantly, this may represent a functionally different region to ventral insula (and perhaps orbitofrontal) cortical areas that may preferentially represent decreases in sympathetic arousal or enhancement of parasympathetic activity. In particular, such an insula region may represent an integrative substrate for disgust representation to which our present study was insensitive.

At a psychophysiological level, our observation of heart rate enhancement associated with sadness and anger, in contrast to heart rate reductions induced by happiness and disgust, is consistent with earlier studies (Ekman et al., 1983; Levenson et al., 1990). Such studies have typically measured physiological states associated with the induction of different moods (Collet et 
al., 1997; Ekman et al., 1983; Kuniecki et al., 2003; Levenson et al., 1990). A strength of our paradigm, arising from the use of the forewarned reaction-time task, is that we reliably demonstrate significant modulatory influences on orienting heart rate acceleration to brief presentations of emotional stimuli. Moreover, demonstration of differential responses to transient emotional stimuli provides evidence for a contribution to emotional processing of somatic mechanisms proposed in the James-Lange theory (Damasio, 1994; James, 1894). The measurement of autonomic nervous activity across multiple axes further indicates that emotion types may be further differentiated by the pattern of bodily responses. Thus, electrodermal activity, respiration, and facial temperature contribute to distinctive emotion-specific bodily states (Collet et al., 1997; Ekman et al., 1983; Levenson et al., 1990). The phylogenetic basis of enhanced cardiac arousal to perceived expressions of sadness and anger (relative to happiness and disgust) is intriguing, since it neither represents a primary distinction between positive and negative emotions, nor approach/avoidance dimensions of emotion (where expressions of sadness may induce positive behavioral approach relative to anger and disgust). Moreover, our neuroimaging evidence suggests that the functional resources of many sensory, associative, and autonomic brain regions are recruited in the dissociation of angry and sad from happy and disgusted expressions. This distinction therefore may represent an important dimension of "Jamesian" processing of emotions, where perceived emotions in others automatically induce changes in the bodily state of the observer that mirror empathetically the bodily autonomic responses accompanying subjective experience of discrete mood states (Ekman et al., 1983). The possibility that medial orbitofrontal activity serves to dampen sympathetic arousal responses to emotional faces (e.g., during happy or disgust processing) is suggested by the inverse relationship between activity in this region and predicted cardiac acceleration. This observation is consistent with evidence for inverse relationships between subgenual activity and orienting or sympathetic electrodermal responses, perhaps suggesting an antisympathetic or parasympathetic role (Gusnard et al., 2001; Nagai et al., 2004). Moreover, medial orbitofrontal activity has been associated with processing of positive or rewarding face stimuli (O'Doherty et al., 2003). However, orbitofrontal signal dropout in adjacent cortex and relatively low effect size limit our further interpretation of activity in this region.

Our exploration of the mechanisms predicted by the JamesLange theory was constrained by our use of only one (cardiac) axis of autonomic arousal and emphasis away from subjective feeling states. The lack of difference in cardiac arousal between sad and disgust stimulus processing may reflect differences in cardiac sympathetic/parasympathetic activity and balance that are not reflected in overall heart rate changes. Moreover, while we identified brain areas that mediate emotional production of autonomic arousal, we also highlighted the presence of discriminatory responses in caudate and left insula regions that were apparently independent of our cardiac index of autonomic arousal consistent with the presence of parallel non-Jamesian mechanisms that may support adaptive emotional processing without predicting or relying on differential, interpretable changes in peripheral arousal state (Critchley et al., 2001, 2002). Such mechanisms can be embodied within with a multicomponent model of emotion in which interactions between component processes permit adaptive responses, for example, the volitional control of emotion.

To summarize, the James-Lange theory of emotion predicts a mechanism by which emotive stimuli may automatically elicit differential changes in bodily arousal state that feedback centrally to influence the emotional interpretation of the stimuli. We demonstrate that presentation of different emotional facial expressions is sufficient to differentially modulate autonomic control of the heart, wherein angry and sad expressions enhance heart rate acceleration relative to happiness and disgust. We also show that brain activity in regions implicated in visual processing of facial expressions and regions implicated in emotional learning and autonomic control predicts the cardiac response evoked by emotional faces. These observations highlight the central mechanisms through which emotive stimuli may produce differential bodily responses, a critical mechanism necessary for differential patterns of bodily arousal, as predicted in the James-Lange theory, to contribute to emotional color engendered by 'exciting objects' (James, 1894).

\section{Acknowledgments}

This work was supported by a Wellcome Clinician Scientist Fellowship to HDC and a Programme Grant to RJD. We acknowledge the assistance and helpful discussions of members of the Emotion Group at the Wellcome Department of Imaging Neuroscience.

\section{References}

Adolphs, R., Tranel, D., Damasio, H., Damasio, A., 1994. Impaired recognition of emotion in facial expressions following bilateral damage to the human amygdala. Nature 372, 669-672.

Barbas, H., Saha, S., Rempel-Clower, N., Gashghaei, T., 2003. Serial pathways from the primate prefrontal cortex to autonomic areas may influence emotional expression. BMC Neurosci. 4, 25.

Barrera, M.E., Maurer, D., 1981. The perception of facial expressions by the three-month-old. Child Dev. 52, 203-206.

Baylis, L.L., Gaffan, D., 1991. Amygdalectomy and ventromedial prefrontal ablation produce similar deficits in food choice and in simple object discrimination learning for an unseen reward. Exp. Brain Res. 86, $617-622$.

Blair, R.J., 2003. Facial expressions, their communicatory functions and neuro-cognitive substrates. Philos. Trans. R. Soc. London, B Biol. Sci. $358,561-572$

Breiter, H.C., Etcoff, N.L., Whalen, P.J., Kennedy, W.A., Rauch, S.L., Buckner, R.L., Strauss, M.M., Hyman, S.E., Rosen, B.R., 1996. Response and habituation of the human amygdala during visual processing of facial expression. Neuron 17, 875-887.

Buchel, C., Morris, J., Dolan, R.J., Friston, K.J., 1998. Brain systems mediating aversive conditioning: an event-related fMRI study. Neuron 20, 947-957.

Cannon, W.B., 1927. The James-Lange theory of emotions. Am. J. Psychol. $39,115-1124$

Collet, C., Vernet-Maury, E., Delhomme, G., Dittmar, A., 1997. Autonomic nervous system response patterns specificity to basic emotions. J. Auton. Nerv. Syst. 62, 45-57.

Critchley, H.D., Corfield, D.R., Chandler, M.P., Mathias, C.J., Dolan, R.J., 2000a. Cerebral correlates of autonomic cardiovascular arousal: a functional neuroimaging investigation. J. Physiol. (London) 523, $259-270$.

Critchley, H.D., Elliot, R., Mathias, C.J., Dolan, R.J., 2000b. Neural activity 
relating to the generation and representation of galvanic skin conductance response: a functional magnetic imaging study. J. Neurosci. 20, 3033-3040.

Critchley, H.D., Mathias, C.J., Dolan, R.J., 2001. Neural correlates of first and second-order representation of bodily states. Nat. Neurosci. 2, 207-212.

Critchley, H.D., Mathias, C.J., Dolan, R.J., 2002. Fear-conditioning in humans: the influence of awareness and arousal on functional neuroanatomy. Neuron 33, 653-663.

Critchley, H.D., Josephs, O., O’Doherty, J., Zanini, S., Dewar, B.-K., Mathias, C.J., Cipolotti, L., Shallice, T., Dolan, R.J., 2003. Human cingulate cortex and autonomic cardiovascular control: converging neuroimaging and clinical evidence. Brain 216, 2139-2152.

Critchley, H.D., Wiens, S., Rotshtein, P., Ohman, A., Dolan, R.J., 2004. Neural systems supporting interoceptive awareness. Nat. Neurosci. 7 , $189-195$.

Damasio, A.R., 1994. Descartes' Error: Emotion, Reason and the Human Brain. Grosset/Putnam, New York.

Deichmann, R., Gottfried, J.A., Hutton, C., Turner, R., 2003. Optimized EPI for fMRI studies of the orbitofrontal cortex. NeuroImage 19, $430-441$.

Dimberg, U., Thunberg, M., Elmehed, K., 2000. Unconscious facial reactions to emotional facial expressions. Psychol. Sci. 11, 86-89.

Dolan, R.J., 2002. Emotion, cognition, and behavior. Science 298, $1191-1194$.

Duvernoy, H.M., 1999. The Human Brain, second ed. Springer, Wien New York.

Ekman, P., 1993. Facial expression and emotion. Am. Psychol. 48, 384-392.

Ekman, P., Levenson, R.W., Friesen, W.V., 1983. Autonomic nervous system activity distinguishes among emotions. Science 221, 1208-1210.

Frith, C.D., Allen, H.A., 1983. The skin conductance orienting response as an index of attention. Biol. Psychol. 17, 27-39.

Friston, K.J., Glaser, D.E., Henson, R.N., Kiebel, S., Phillips, C., Ashburner, J., 2002. Classical and Bayesian inference in neuroimaging: applications. NeuroImage 16, 484-512.

Gottfried, J.A., O'Doherty, J., Dolan, R.J., 2003. Encoding predictive reward value in human amygdala and orbitofrontal cortex. Science 301, 1104-1107.

Gundel, H., O'Connor, M.F., Littrell, L., Fort, C., Lane, R.D., 2003. Functional neuroanatomy of grief: an fMRI study. Am. J. Psychiatry $160,1946-1953$.

Gusnard, D.A., Akbudak, E., Shulman, G.L., Raichle, M.E., 2001. Medial prefrontal cortex and self-referential mental activity: relation to a default mode of brain function. Proc. Natl. Acad. Sci. U. S. A. 27, $4259-4264$

Hamann, S., Mao, H., 2002. Positive and negative emotional verbal stimuli elicit activity in the left amygdala. NeuroReport 13, 15-19.

Hamann, S.B., Stefanacci, L., Squire, L.R., Adolphs, R., Tranel, D., Damasio, H., Damasio, A., 1996. Recognizing facial emotion. Nature 379, 497.

Hasselmo, M.E., Rolls, E.T., Baylis, G.C., 1989. The role of expression and identity in the face-selective responses of neurons in the temporal visual cortex of the monkey. Behav. Brain Res. 32, 203-218.

Iwata, J., Chida, K., LeDoux, J.E., 1987. Cardiovascular responses elicited by stimulation of neurons in the central amygdaloid nucleus in awake but not anesthetized rats resemble conditioned emotional responses. Brain Res. 418, 183-188.

James, W., 1894. Physical basis of emotion. Psychol. Rev. 1, 516-529 (reprinted in 1994. Psychological Rev. 101, 205-210).

Jennings, J.R., van der Molen, M.W., Debski, K.B., 2002. Probing the response selection bottleneck with a cardiac measure: individual differences in strategy for a psychological refractory period task. Biol. Psychol. 59, 207-230.

Josephs, O., Deichmann, R., Turner, R., 2000. Trajectory measurement and generalised reconstruction in rectilinear EPI. NeuroImage 11, S543.

Kanwisher, N., McDermott, J., Chun, M.M., 1997. The fusiform face area: a module in human extrastriate cortex specialized for face perception. J. Neurosci. 17, 4302-4311.

Kuniecki, M., Urbanik, A., Sobiecka, B., Kozub, J., Binder, M., 2003. Central control of heart rate changes during visual affective processing as revealed by fMRI. Acta Neurobiol. Exp. Wars. 63, 39-48.

Kuskowski, M.A., Pardo, J.V., 1999. The role of the fusiform gyrus in successful encoding of face stimuli. NeuroImage 9, 599-610.

Lang, P.J., Bradley, M.M., Cuthbert, B.N., 1998. Emotion, motivation, and anxiety: brain mechanisms and psychophysiology. Biol. Psychiatry 44, $1248-1263$.

LeDoux, J., 1996. The Emotional Brain: The Mysterious Underpinnings of Emotional Life. Simon and Schuster, New York

LeDoux, J.E., Iwata, J., Cicchetti, P., Reis, D.J., 1998. Different projections of the central amygdaloid nucleus mediate autonomic and behavioral correlates of conditioned fear. J. Neurosci. 8, 2517-2529.

Levenson, R.W., Ekman, P., Friesen, W.V., 1990. Voluntary facial action generates emotion-specific autonomic nervous system activity. Psychophysiology 27, 363-384.

Lundqvist, D., Flykt, A., Öhman, A., 1998. The Karolinska Directed Emotional Faces-KDEF, CD ROM from Department of Clinical Neuroscience, Psychology section, Karolinska Institutet, ISBN 91630-7164-9.

Morris, J.S., Frith, C.D., Perrett, D.I., Rowland, D., Young, A.W., Calder, A.J., Dolan, R.J., 1996. A differential neural response in the human amygdala to fearful and happy facial expressions. Nature $383,812-815$.

Morris, J.S., Friston, K.J., Dolan, R.J., 1997. Neural responses to salient visual stimuli. Proc. R. Soc. London, B Biol. Sci. 264, 769-775.

Morris, J.S., Friston, K.J., Buchel, C., Frith, C.D., Young, A.W., Calder, A.J., Dolan, R.J., 1998. A neuromodulatory role for the human amygdala in processing emotional facial expressions. Brain $121,47-57$

Morris, J.S., deBonis, M., Dolan, R.J., 2002. Human amygdala responses to fearful eyes. NeuroImage 17, 214-222.

Nagai, Y., Critchley, H.D., Featherstone, E., Trimble, M.R., Dolan, R.J., 2004. Activity in ventromedial prefrontal cortex covaries with sympathetic skin conductance level: a physiological account of a "default mode" of brain function. NeuroImage 22, 243-251.

O’Doherty, J.P., Deichmann, R., Critchley, H.D., Dolan, R.J., 2002. Neural responses during anticipation of a primary taste reward. Neuron 33 , $815-826$

O’Doherty, J.P., Winston, J., Critchley, H., Perrett, D., Burt, D.M., Dolan, R.J., 2003. Beauty in a smile: the role of medial orbitofrontal cortex in facial attractiveness. Neuropsychologia 41, 147-155.

Phelps, E.A., O'Connor, K.J., Gatenby, J.C., Gore, J.C., Grillon, C., Davis, M., 2001. Activation of the left amygdala to a cognitive representation of fear. Nat. Neurosci. 4, 437-441.

Phillips, M.L., Young, A.W., Senior, C., Brammer, M., Andrew, C., Calder, A.J., Bullmore, E.T., Perrett, D.I., Rowland, D., Williams, S.C., Gray, J.A., David, A.S., 1997. A specific neural substrate for perceiving facial expressions of disgust. Nature 389, 495-498.

Puce, A., Allison, T., Bentin, S., Gore, J.C., McCarthy, G., 1998. Temporal cortex activation in humans viewing eye and mouth movements. J. Neurosci. 18, 2188-2199.

Schachter, S., Singer, J.E., 1962. Cognitive, social and physiological determinants of emotional state. Psychol. Rev. 69, 379-399.

Schwartz, C.E., Wright, C.I., Shin, L.M., Kagan, J., Whalen, P.J., McMullin, K.G., Rauch, S.L., 2003. Differential amygdalar response to novel versus newly familiar neutral faces: a functional MRI probe developed for studying inhibited temperament. Biol. Psychiatry 53, $854-862$

Surakka, V., Hietanen, J.K., 1998. Facial and emotional reactions to Duchenne and non-Duchenne smiles. Int. J. Psychophysiol. 29, 23-33.

Vuilleumier, P., Armony, J.L., Driver, J., Dolan, R.J., 2001. Effects of attention and emotion on face processing in the human brain: an eventrelated fMRI study. Neuron 30, 829-841.

Williams, L.M., Phillips, M.L., Brammer, M.J., Skerrett, D., Lagopoulos, 
J., Rennie, C., Bahramali, H., Olivieri, G., David, A.S., Peduto, A., Gordon, E., 2001. Arousal dissociates amygdala and hippocampal fear responses: evidence from simultaneous fMRI and skin conductance recording. NeuroImage 14, 1070-1079.

Winston, J.S., O’Doherty, J., Dolan, R.J., 2003. Common and distinct neural responses during direct and incidental processing of multiple facial emotions. NeuroImage 20, 84-97.

Winston, J.S., Henson, R.N., Fine-Goulden, M.R., Dolan, R.J., 2004. fMRI-adaptation reveals dissociable neural representations of identity and expression in face perception. J. Neurophysiol. 92, 1830-1839. 